\title{
Hemodialysis-associated amyloidosis with cervical spinal cord compression and incomplete tetraplegia: a case report
}

\author{
S Moslavac*,1, I Dzidic ${ }^{1}, Z$ Kejla ${ }^{2}$ and D Tomas ${ }^{3}$ \\ ${ }^{1}$ Spinal Unit, Special Medical Rehabilitation Hospital, Varazdinske Toplice, Croatia; ${ }^{2}$ Department for Spinal Surgery, \\ Traumatology Clinic, Zagreb, Croatia; ${ }^{3}$ Ljudevit Jurak Department of Pathology, Sisters of Charity University \\ Hospital, Zagreb, Croatia
}

\begin{abstract}
Study design: Case report.
Objectives: To report a rare case of hemodialysis-associated amyloidosis of cervical spinal canal with incomplete tetraplegia in long-term hemodialysis patient.

Settings: Traumatology Clinic, Zagreb; Spinal Unit, Varazdinske Toplice, Croatia.

Methods: Forty-seven-year-old male patient at long-term hemodialysis treatment developed progressive limbs weakness, graded as C4 ASIA C. Cervical computed tomography myelography showed extreme narrowing of the spinal canal. Decompressive laminectomy with bilateral foraminotomies was performed and histological examination revealed amyloidal deposition.

Results: Rehabilitation program started immediately after surgery with physical and occupational therapy, along with psychological support aiming at restoring full activities of daily living. Three months after surgery, the patient returned to his community, neurologically improved to ASIA E. Hemodialysis program was regularly maintained perioperatively and during the rehabilitation.

Conclusion: Vertebral involvement with neurological deterioration in hemodialysis-associated amyloidosis patients prompts for early diagnosis, surgical and rehabilitation management, in this instance with good outcome.

Spinal Cord (2007) 45, 799-801; doi:10.1038/sj.sc.3102112; published online 28 August 2007
\end{abstract}

Keywords: hemodialysis-associated amyloidosis; tetraplegia; rehabilitation

\section{Introduction}

Hemodialysis-associated amyloidosis became a frequent and sometimes disabling complication in long-term hemodialysis patients, with deposition of amyloidal fibrils containing $\beta$-2-microglobulin in collagen tissue, particularly in the synovia of joints and tendons, and in subchondral bone. Most common clinical manifestations are carpal tunnel syndrome and arthropathy of shoulders, knees, hips and axial skeleton, possibly leading to the neurological compromise. Combination of disc space narrowing, vertebral body erosion and pseudocysts formation, in the presence of polyarthropathy, chronic renal failure and carpal tunnel syndrome, suggests the diagnosis of destructive spondyloarthropathy, which should be proved by the histological finding of $\beta$-2-microglobulin - amyloid. ${ }^{1}$

*Correspondence: S Moslavac, Spinal Unit, Special Medical Rehabilitation Hospital, 42223 Varazdinske Toplice, Croatia

\section{Case report}

We present 47-year-old middle-eastern European male patient with chronic renal insufficiency and a history of 22 years at hemodialysis treatment, who developed progressive limbs weakness in 2-month period. He already had been operated for bilateral carpal tunnel syndrome caused by amyloidal depositions and had unsuccessful kidney transplantation 1 year after commencing hemodialysis. Previously, he acquired hepatitis $\mathrm{B}$ and $\mathrm{C}$ infections, had a stroke without residual neurological deficit and had subtotal parathyroidectomy. Initial neurological examination showed reduced muscle strength of all key muscles with grade 3 and preserved sensory, thus graded as C4 ASIA C. Cervical computed tomography (CT) myelography showed extreme narrowing of the spinal canal, with 7-mm thick posterior longitudinal ligament and $4-5 \mathrm{~mm}$ width of spinal cord in $\mathrm{C} 1-\mathrm{C} 7$ region (Figure 1). Decompressive laminectomy from $\mathrm{C} 3$ to $\mathrm{C} 7$, with extension to partial 

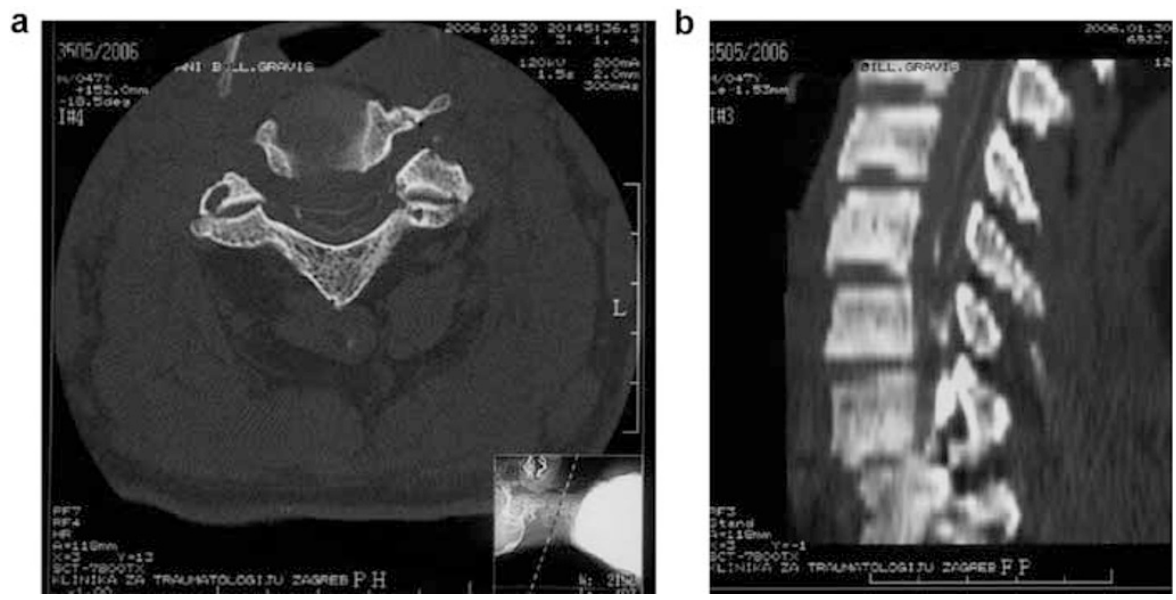

Figure 1 CT myelography showing narrowing of the spinal canal, with $7 \mathrm{~mm}$ thick posterior longitudinal ligament and 4-5 mm width of spinal cord in $\mathrm{C} 1-\mathrm{C} 7$ region ((a) axial view, (b) sagittal view)
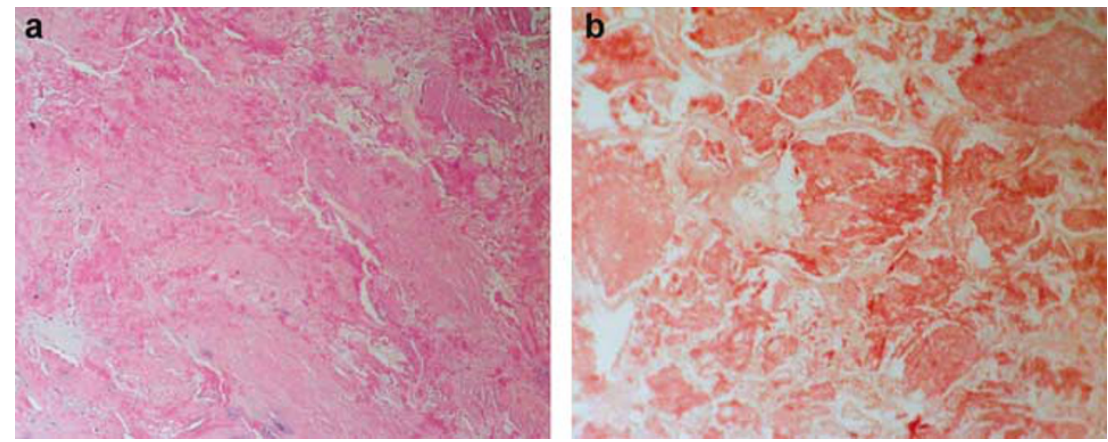

Figure 2 (a) Histological examination revealed amorphous pink deposits, which surrounded and destroyed the trapped native cells of posterior longitudinal ligament $(\mathrm{H} \& \mathrm{E}$, original magnification $\times 100)$. (b) Diagnosis of amyloidosis is confirmed with Congo red stain $($ Congo red, original magnification $\times 100)$

laminectomy of $\mathrm{T} 1$, and bilateral foraminotomies in C3-C7 levels were performed. Histological examination revealed amyloidal depositions both in the superficial and deep layers of posterior longitudinal ligament (Figure 2a). Diagnosis of amyloidosis is confirmed with Congo red stain, which showed green birefringence under polarized light (Figure 2b). Philadelphia collar was applied postoperatively and rehabilitation program started immediately following surgery. The patient was transferred to Spinal Unit of Rehabilitation Hospital at 14th postoperative day. Respiratory therapy consisted of forced voluntary expirations, assisted cough and postural drainage. Kinesiotherapy included passive range of motion exercises to prevent contractures, followed by active isometric exercises to gain muscle power. It enabled the patient to achieve verticalization in braces, while pool hydrotherapy enhanced stability, flexibility and posture. First steps within a pool were made 10 days after the transfer to Spinal Unit and the patient was soon able to support his body weight that allowed ambulation with the use of a walker, cane and finally without aid. Occupational therapy was included to improve hands' functions impaired previously by carpal tunnel pathology as well as cervical compression. Full ADL (activities of daily living) were encouraged and psychological support proved valuable in mastering obstacles imposed by physical handicap. Hemodialysis program was regularly maintained in the preoperative and postoperative period and during rehabilitation.

Three months after surgery, rehabilitation program concluded and our patient returned to his community, independent in ADL, walking without aid, graded as WISCI 20 (Walking Index for Spinal Cord Injury) and neurologically improved to ASIA E.

\section{Discussion}

Clinicians should be aware of hemodialysis-associated amyloidosis syndrome in long-term hemodialysis patients with incidence reported to be between 9 and $18 \% .^{2}$ Sporadically, there are reports about patients with axial amyloidal involvement resulting in neurological impairment. ${ }^{3}$ CT myelography or magnetic resonance imaging clears the diagnosis, above all in cases where there was uncertainty about possible vertebral osteomyelitis formation. ${ }^{4}$ Correctly planned operative 
and rehabilitation effort in our patient resulted in almost complete neurological recovery, although some authors referred better results with anterior decompression and fixation in similar cases. ${ }^{5}$ The axial skeleton involvement with cervical spinal cord compression symptoms was superimposed to already existent impairment of hands' functions caused by bilateral amyloidal depositions in carpal tunnels, proving occupational therapy critical in ADL training. Ambulation was achieved through standardized yet individually tailored program with much time 'lost', because of regular, three times weekly hemodialysis and diminished capacity resulting from chronic anemia (hemoglobin 100-110 g/l) and metabolic disturbances, particularly increased creatinine level $(\geqslant 700 \mu \mathrm{mol} / \mathrm{l})$ in periods before hemodialysis, that accompany renal insufficiency. Calcium $(2.5 \mathrm{mmol} / \mathrm{l})$ and phosphate $(1.15 \mathrm{mmol} / \mathrm{l})$ levels were maintained within normal ranges, with PTH level $4.1 \mathrm{pmol} / 1$, normal but not sufficient, especially as we know that in hemodialysis patient it should be 2.5 times higher than normal values. Fortunately, the patient was motivated for program and did not suffer with typical complications seen in SCI rehabilitation; therefore, we had not seen any setbacks during his rehabilitation. Almost complete neurological and functional recovery and his return to the community represent properly orchestrated diagnostic, surgical and rehabilitative efforts among several facilities. This case report should attain two goals: to raise awareness of this rare complication and to show that neurological deterioration in hemodialysisassociated amyloidosis patients prompts for early diagnosis, surgical and rehabilitation management.

\section{References}

1 Kunz D, Naveau B, Bardun T, Drueke T, Treves R, Dryll A. Destructive spondyloarthropathy in hemodialyzed patients - a new syndrome. Arthritis Rheum 1984; 27: 369-375.

2 Cuffe MJ, Hadley MN, Herrera GA, Morawettz RB. Dialysis-associated spondylo-arthropathy. $J$ Neurosurg 1994; 80: 694-700.

3 Nokura K et al. Dialysis-related spinal canal stenosis: a clinicopathological study on amyloid deposition and its AGE modification. J Neurol Sci 2000; 178: 114-123.

4 Cobby MJ, Adler RS, Swartz R, Martel W. Dialysis-related amyloid arthropathy: MR findings in four patients. Am J Radiology 1991; 157: 1023-1027.

5 Kumar A, Leventhal MR, Freedman EL, Coburn J, Delamarter R. Destructive spondyloarthropathy of the cervical spine in patients with chronic renal failure. Spine 1997; 22: 573-578. 